\title{
The Use of 3D Bodies in a 2D Computerised and Immersive Virtual Reality Body Image Intervention
}

\author{
Nadia MAALIN*1, Kamila R. IRVINE ${ }^{1}$, Andrew IRVINE', \\ Piers L. CORNELISSEN ${ }^{2}$, Kay L. RITCHIE ${ }^{1}$ and Martin J. TOVÉE ${ }^{1}$ \\ ${ }^{1}$ School of Psychology, University of Lincoln, Lincoln, England; \\ 2 School of Psychology, Northumbria University, Newcastle Upon Tyne, England
}

https://doi.org/10.15221/19.142

\begin{abstract}
Body image disturbances are associated with eating disorders such as Anorexia Nervosa and Bulimia Nervosa, supporting the importance of developing interventions that specifically target body image. Most interventions focus on targeting sociocultural aspects of body image as opposed to the perceptual component. Here a perceptual body image intervention was employed, based on categorical judgements of body size ('thin' or 'fat') following an existing cognitive bias modification technique. Previously, this intervention, has been piloted in 2D in females with heightened body concerns and Anorexia Nervosa. Findings suggest that by using inflationary feedback the program can modify categorical thin-fat body judgements, with improvements in psychological self-report measurements, and these changes were maintained at follow-up. The 3D bodies were computer-generated, calibrated based on body mass index, and presented in $2 \mathrm{D}$ in the computerised intervention. In the current work we present this intervention delivered in 2D and in 3D virtual reality (VR). Using VR allows for life-sized (scaled 1:1), volumetric presentations of 3D body stimuli in an immersive environment. While further research of this intervention technique is needed, preliminary findings suggest that it may be a beneficial addition to body image treatment.
\end{abstract}

Keywords: body image, virtual reality, body image intervention, 3D human figures, body size perception.

\section{Introduction}

\subsection{Body image distortion (BID)}

Body image is comprised of two distinct yet interrelated components: attitudinal/cognitive-affective component - thoughts and feelings about ones' own body, and a perceptual component - a mental picture of one's own body [1, 2, 3]. Body image has been found to influence day-to-day functioning, mental health, psychopathology (e.g. feelings of shame, depression, and anxious thoughts [4]), selfesteem, social interactions and quality of life $[5,6,7]$.

Disturbance of the perceptual component, body image distortion (BID) is the discrepancy between ones' actual body size and the perception of their own body size. This may be inaccurate, for example Anorexic patients are thought to over-estimate their own size [1, 8, 9]. Currently, body image distortion is considered to be a cardinal feature of Anorexia Nervosa, and is included as one of the diagnostic criteria of the disorder: "disturbance in the way in which one's body weight or shape is experienced" [10]. Overall, treatment of $A N$ is difficult and relapse is a significant problem, recent review of body image-specific interventions concluded that their effectiveness is promising and highlighted the need for development of new psychological interventions [11].

\subsection{The development of a body size perception intervention}

Two recent studies of computer-based interventions have shown that the perceptual boundary between 'thin' and 'fat' bodies can be manipulated experimentally. Adapted from an emotion recognition bias training programme by Penton-Voak et al., [12] Gledhill et al. [13] used the technique to increase participants' subjective categorical boundaries for thin-fat bodies towards fatter bodies in samples of females with heightened body image concerns and Anorexia Nervosa. The effect was maintained at follow up and accompanied by self-reported improvements in psychological concerns.

*nmaalin@lincoln.ac.uk 
A similar study by Szostak [14] using a similar body size perception training task to encourage the interpretation of thinness over heaviness of bodies in the normal BMI range. The intervention significantly modified the categorical boundary for up to two-weeks, and was associated with improvements in body image and disordered eating. Furthermore, there were changes in the selection of the most attractive and ideal body size, which was higher in BMI post-training.

Together, these findings suggest that this training program may be a useful technological intervention that can be used in females with heightened body concerns and eating disorder patients as a technique to alter judgements of body size, with corresponding improvements in psychological concerns. Further investigation into the replicability and effectiveness of this perceptual intervention is needed to gain a better insight into the clinical effectiveness and utility as a therapeutic tool.

Therefore, the aim of this work is to discuss the use of 3D technology in this body size perception intervention. Firstly, the replicability of the computerised intervention using 3D computer-generated bodies will be discussed (data collection on-going). Secondly, the effectiveness of delivering the intervention in immersive virtual reality (VR) using life-sized, volumetric bodies will be evaluated in comparison to the original computerised intervention by Gledhill et al. [13].

\section{Method}

\subsection{Participants}

To date, 33 females took part in the computerised study and 60 participants took part in the VR study. All females were screened using the 16-item Body Shape Questionnaire [15] to select those with heightened body, shape and weight concerns, but no history or current diagnosis of an eating disorder.

\subsection{Stimulus creation}

\subsubsection{Computer-generated images for use in the computerized intervention}

The female body stimuli used in this intervention were created using a 3D modelling Software, Daz Studio. To generate the stimuli, a base model was created based on Health Survey for England 2008 dataset in order to create a model that would resemble the average body shape and height of a 25 year old Caucasian female. The body was manipulated in body size using full-body adiposity morphs to create a range body sizes from underweight to obese. The BMI of the models was computed using a calibration equation that predicted BMI from waist and hip circumferences, age, sex, height and ethnicity in the HSE database [16]. The final set of 15 bodies varying in size from 15.36 to $33.56 \mathrm{BMI}$ units were selected based on pilot studies by Gledhill [17] and rendered for use in this intervention.

\subsubsection{Stimulus creation for use in the VR intervention}

A base model provided from the Manuel Bastioni Laboratory was used to develop a set of 15 3D models in Unreal Engine v4.18, ranging from 15.45 to $33.70 \mathrm{BMI}$ units, following the same calibration equation used above.

\subsection{Body size perception training task}

The task was comprised of two phases: baseline and training. In both phases, participants were presented with a body model and asked to respond whether they consider the stimulus to be 'thin' or 'fat'. In the baseline phase, the response triggers the presentation of the next model. The baseline allows for calculation of the thin-fat categorical boundary for that participant and was taken on each day of the study. In the training phase, the decision was followed by feedback, e.g. 'Incorrect! That body was thin'. In the intervention group, the feedback was inflationary, it was designed to shift participants' categorical boundary by two bodies further along the sequence based on their baseline categorical boundary for the day. In the control group, participants received feedback that was consistent with their baseline categorical boundary in order to reinforce their perceptual thin-fat judgements. The training phase was ran on four consecutive days.

\subsection{Cognitive-affective measurements}

A set of validated questionnaires assessing body image, disordered eating, depressive symptoms and self-esteem were used: The Eating Disorders Examination Questionnaire (EDE-Q; [18]), Rosenberg Self-Esteem Scale (RSE; [19]), Beck Depression Inventory (BDI; [20]) and the 16-item Body Shape Questionnaire (BSQ-16b; [15]). The questionnaires were completed at day 1 (a baseline measurement), at day 4 (immediately after training) and at the follow-up date on day 14.

\subsection{Body measurements}

The participants actual BMI (weight $(\mathrm{kg}) /[\text { height }(\mathrm{m})]^{2}$ ) was measured using a calibrated scale and a stadiometer. 


\section{Data analysis}

Preliminary data analysis is currently on-going for this project. The data will be analysed using linear mixed effect models to assess whether the intervention does produce a shift in categorical boundary and whether there are any changes in psychological concerns.

\section{Discussion}

Whilst the clinical effectiveness and therapeutic utility of this training program warrants further investigation, the use of a technologically delivered intervention is an exciting, new approach to treating body image in females with heightened body concerns. This approach combines a cognitive bias modification technique with modern 3D technology to create a training program which could be a useful tool for manipulating body size perception and improving psychological concerns. This technological approach opens new opportunities to incorporate 3D and VR technology into the psychological treatment of body image and disordered eating, which is currently an expanding area of research [21, 22]. The extension into VR may be a beneficial improvement as it allows for life-sized (scaled 1:1) presentations of $3 \mathrm{D}$ bodies in an immersive environment which adds to the ecological validity and interactive nature of the intervention. In the future, this program could be delivered as additional treatment alongside existing therapeutic tools. This does require the use of technology (a computer screen/laptop) and some more advanced technical equipment (if delivered in virtual reality), however, should the replicability and clinical effectiveness of this intervention be proven, the relative low cost of the technical equipment would not be prohibitive for use in clinical and research settings.

\section{References}

[1] T. F. Cash and E. A. Deagle, "The nature and extent of body-image disturbances in anorexia nervosa and bulimia nervosa: A meta-analysis," Int. J. Eat. Disord., vol. 22, (2), pp. 107-126, 1997.

[2] P. D. Slade, "Body image in anorexia nervosa," The British Journal of Psychiatry, vol. 153, (S2), pp. 20-22, 1988.

[3] P. Schilder, The Image and Appearance of the Human Body. New York: International Universities Press.,1935.

[4] Mental Health Foundation, Body Image: How we think and feel about our bodies. London: Mental Health Foundation, May 13, 2019. Accessed on: August $18^{\text {th }}, 2019$. Available: https://www.mentalhealth.org.uk/publications/body-image-report.

[5] D. M. Garner, "The 1997 body image survey results," Psychology Today, vol. 30, (1), pp. 30-44, 1997.

[6] S. Griffiths et al, "Sex differences in the relationships between body dissatisfaction, quality of life and psychological distress," Aust. N. Z. J. Public Health, vol. 40, (6), pp. 518-522, 2016.

[7] $\mathrm{H}$. Sharpe et al, "The relative importance of dissatisfaction, overvaluation and preoccupation with weight and shape for predicting onset of disordered eating behaviors and depressive symptoms over 15 years," Int. J. Eat. Disord., 2018.

[8] K. K. Cornelissen et al, "The influence of personal BMI on body size estimations and sensitivity to body size change in anorexia spectrum disorders," Body Image, vol. 13, pp. 75-85, 2015.

[9] D. Benninghoven et al, "Body images of patients with anorexia nervosa, bulimia nervosa and female control subjects: a comparison with male ideals of female attractiveness," Body Image, vol. 4, (1), pp. 51-59, 2007.

[10] American Psychiatric Association, Diagnostic and Statistical Manual of Mental Disorders (DSM5®). American Psychiatric Pub, 2013.

[11] K. Ziser et al, "Effectiveness of body image directed interventions in patients with anorexia nervosa: A systematic review," Int. J. Eat. Disord., vol. 51, (10), pp. 1121-1127, 2018.

[12] I. S. Penton-Voak et al, "Increasing recognition of happiness in ambiguous facial expressions reduces anger and aggressive behavior," Psychological Science, vol. 24, (5), pp. 688-697, 2013.

[13] L. J. Gledhill et al, "An interactive training programme to treat body image disturbance," British Journal of Health Psychology, vol. 22, (1), pp. 60-76, 2017.

[14] N. M. Szostak, Negative Body Image and Cognitive Biases to Body Size, 2018.

[15] C. Evans and B. Dolan, "Body Shape Questionnaire: derivation of shortened "alternate forms"," Int. J. Eat. Disord., vol. 13, (3), pp. 315-321, 1993. 
[16] K. Cornelissen, What does it Mean to have Distorted Body Image in Anorexia Nervosa?, 2016.

[17] L. J. Gledhill, "Investigating preferences for body size, and developing a program to modify distorted body size perception." 2015.

[18] C. G. Fairburn and S. J. Beglin, "Assessment of eating disorders: interview or self-report questionnaire?" Int. J. Eat. Disord., vol. 16, (4), pp. 363, 1994.

[19] M. Rosenberg, "Rosenberg self-esteem scale (RSE)," Acceptance and Commitment Therapy. Measures Package, vol. 61, pp. 52, 1965.

[20] A. Beck et al, "An inventory for measuring depression," Arch. Gen. Psychiatry, vol. 4, pp. 561-571, 1961.

[21] M. Ferrer-Garcia, J. Gutiérrez-Maldonado and G. Riva, "Virtual reality based treatments in eating disorders and obesity: a review," Journal of Contemporary Psychotherapy, vol. 43, (4), pp. 207$221,2013$.

[22] A. Koskina, I. C. Campbell and U. Schmidt, "Exposure therapy in eating disorders revisited," Neuroscience \& Biobehavioral Reviews, vol. 37, (2), pp. 193-208, 2013. 